\title{
The Impact of Positional Encodings on Multilingual Compression
}

\author{
Vinit Ravishankar \\ Language Technology Group \\ University of Oslo \\ Norway \\ vinitreifi.uio.no
}

\author{
Anders Søgaard \\ Department of Computer Science \\ University of Copenhagen \\ Denmark \\ soegaardedi.ku.dk
}

\begin{abstract}
In order to preserve word-order information in a non-autoregressive setting, transformer architectures tend to include positional knowledge, by (for instance) adding positional encodings to token embeddings. Several modifications have been proposed over the sinusoidal positional encodings used in the original transformer architecture; these include, for instance, separating position encodings and token embeddings, or directly modifying attention weights based on the distance between word pairs. We first show that surprisingly, while these modifications tend to improve monolingual language models, none of them result in better multilingual language models. We then answer why that is: Sinusoidal encodings were explicitly designed to facilitate compositionality by allowing linear projections over arbitrary time steps. Higher variances in multilingual training distributions requires higher compression, in which case, compositionality becomes indispensable. Learned absolute positional encodings (e.g., in mBERT) tend to approximate sinusoidal embeddings in multilingual settings, but more complex positional encoding architectures lack the inductive bias to effectively learn compositionality and cross-lingual alignment. In other words, while sinusoidal positional encodings were originally designed for monolingual applications, they are particularly useful in multilingual language models.
\end{abstract}

\section{Introduction}

Multiple recent papers have attempted to pinpoint precisely what components of multilingual language models enable cross-lingual transfer. Pires et al. (2019) show that although wordpiece overlap tends to improve cross-lingual transfer performance, even languages with different scripts (and no shared subwords) may enable zero-shot transfer. Wu and Dredze (2019) report similar results on a wider range of tasks. Artetxe et al. (2020) show

\begin{tabular}{lll}
\hline Sinusoidal & See $\S 2$ & Vaswani et al. (2017) \\
Absolute & $\left(\left(w_{i}+p_{i}\right) W^{Q, 1}\right)$ & Devlin et al. (2019) \\
& $\left(\left(w_{j}+p_{j}\right) W^{K, 1}\right)^{\top}$ & \\
TUPE & $\left(x_{i}^{l} W^{Q, l}\right)\left(x_{j}^{l} W^{K, l}\right)^{\top}+$ & Ke et al. (2020) \\
& $\left(p_{i} U^{Q}\right)\left(p_{j} U^{K}\right)^{\top}$ & \\
TUPE(r) & $\ldots+b_{j-i}$ & \\
Relative(k) & $\left(x_{i} W^{Q}\right)\left(x_{j} W^{K}+a_{i j}\right)^{\top}$ & Shaw et al. (2018) \\
Relative(k/q) & $\left(x_{i} W^{Q}+a_{i j}\right)$ & Huang et al. (2020) \\
& $\left(x_{j} W^{K}+a_{i j}\right)^{\top}$ & \\
\hline
\end{tabular}

Table 1: We compare six positional encodings and their impact on cross-lingual generalization in multilingual language models

that neither a shared vocabulary nor joint multilingual pre-training are necessary to train successful multilingual models. K et al. (2020) find that model depth is a contributor to transfer performance, but that reducing the number of self-attention heads does not have much of an effect.

Our starting point is Dufter and Schütze (2021), who claim that a) multilingual compression is caused by forced parameter sharing across languages, and that $b$ ) positional encodings play a significant role in the creation of a multilingual space, even in the absence of shared subwords and shared special tokens, like delimiters.

Contributions We build on Dufter and Schütze (2021) and demonstrate, through a series of experiments on synthetic and real data, that the choice of positional encoding mechanism has a significant effect on cross-lingual model performance: While many positional encodings have been proposed in monolingual settings as improvements over sinusoidal or absolute positional encodings, originally proposed in Vaswani et al. (2017) and Devlin et al. (2019), including untied positional encodings (TUPE; Ke et al. (2020)) and relative positional encodings (Shaw et al., 2018; Huang et al., 2020), none of these better facilitate cross-lingual compression or sharing. In fact, multilingual language models trained with untied or relative positional encodings exhibit much worse cross-lingual perfor- 
mance. We show that this is because sinusoidal embeddings facilitate compositionality, which we argue is particularly important for cross-lingual compression. We present a method for quantifying the compositionality of positional encodings, and find additional evidence for this hypothesis in word-position correlations and ablation studies. We are, to the best of our knowledge, the first to show this asymmetry between monolingual and multilingual language model training. Our experiments rely on the protocols in Dufter and Schütze (2021), but in addition to simple experiments with their Bible data, we also replicate all our experiments on Wikipedia data. Rather than relying on deterministic perturbations of data, as in Dufter and Schütze (2021) and Sinha et al. (2021), we make novel use of Galactic Dependencies (Wang and Eisner, 2016) in our experiments. Based on our experiments, we recommend caution when adopting methods developed for monolingual language models when training multilingual models, as well as that future work on positional encoding mechanisms also provides evaluations in multilingual settings.

\section{Positional encodings}

Positional encodings have been a mainstay of non-autoregressive transformer-based models right since Vaswani et al. (2017) first proposed the transformer architecture. The motivation being that given that transformers ${ }^{1}$ are order-invariant (as opposed recurrent or convolutional networks), there must be some injection of word order into the encoder. Rather than using conventional "embeddings", Vaswani et al. (2017) use fixed sinusoidal position encodings, where each dimension characterises a sinusoidal waveform of a fixed frequency. Specifically, each encoding $p$ is given as:

$$
\begin{aligned}
p_{(\text {pos }, 2 i)} & =\sin \left(\operatorname{pos} / 10000^{2 i / d_{\text {model }}}\right) \\
p_{(\text {pos }, 2 i+1)} & =\cos \left(\operatorname{pos} / 10000^{2 i / d_{\text {model }}}\right)
\end{aligned}
$$

where pos is the position and $i$ is the dimension. They add these encodings to token representations before passing the sum to the first layer of the selfattention mechanism.

Several alternatives to sinusoidal encodings have been proposed since Vaswani et al. (2017). Most multilingual models tend to use BERT-style (Devlin et al., 2019) learnt absolute positional encodings, where a unique vector is learned and assigned

\footnotetext{
${ }^{1}$ Note that we use "transformers" as shorthand for transformer encoders used for masked language modelling.
}

to each position; these vectors are then added to word representations before being passed to the self-attention mechanism.

As an alternative to such position representations, where every position is represented by a unique vector, relative positional encodings have been proposed (Shaw et al., 2018; Huang et al., 2020). Rather than assigning representations to tokens based on their position, relative positional encoding involves assigning representations to position-position pairs; typically, these encodings are calculated separately and added to the attention matrix. We evaluate both the encodings proposed in Shaw et al. (2018) and the encodings proposed in (Huang et al., 2020) in our experiments below.

He et al. (2021) propose eliminating positionposition correlations, and using separate parameters for word and position representations; Wang et al. (2019) propose using dependency trees instead of raw sequential positions. Ke et al. (2020) recommend eliminating the addition operation in BERT-style representations; they argue that wordposition correlations are effectively nil, and that the addition introduces unnecessary noise. We evaluate two untied positional encodings proposed in $\mathrm{Ke}$ et al. (2020) (TUPE). TUPE modifies absolute representations by a) untying word-position correlations; b) using a separate set of parameters for positional attention and c) untying [CLS] tokens from positions.

We refer to recent surveys (Dufter et al., 2021; Wang et al., 2021) for a more detailed treatment of position encoding methods. We provide a summary of our methods in Table 1. $W^{Q, l}$ and $W^{K, l}$ represent the query/key weights for the attention mechanism at some layer $l$, and $a_{i j}$ or $b_{j-i}$ are learnt vectors corresponding to the offset $j-i$. Note that the untied position-position term $\left(p_{i} U^{Q}\right)\left(p_{j} U^{K}\right)^{\top}$ is added at every layer.

The above positional encodings have been introduced in the context of monolingual pretrained language models, and there has been only a limited amount of work addressing the effect of positional encodings on multilingual models. Liu et al. (2020a) find that positional information tends to hurt machine translation, as the encoder learns a word-order bias towards the source languages. ${ }^{2}$

\footnotetext{
${ }^{2}$ The results in Liu et al. (2020a) apply to zero-shot generalization of fine-tuned, task-specific models and not to how multilingual language models are pretrained. In their experiments, they rely on a pretrained language model with absolute positional encodings. In fact, what they show is that freezing
} 
Artetxe et al. (2020) find that language-specific positional representations help in an adapter-based training scenario. Ding et al. (2020) attempt to account for structural differences between languages by using bracketing transduction grammar trees to reorder position labels (and find that it helps). Liu et al. (2020b) find that models that are relatively agnostic to word-order tend to perform better in cross-lingual settings; they hypothesise that large multilingual encoders, being trained on languages with drastic differences in word orders, tend to have order-agnostic positional encodings, and thus discourage fine-tuning positional encodings downstream. Contemporaneous with this work, Sinha et al. (2021) show that positional information is important for monolingual models even given unnatural, randomly shuffled word ordering.

Dufter and Schütze (2021) present a set of experiments training smaller language models on bilingual corpora, consisting of the same corpus in English and "fake-English", which is English with a shifted BPE vocabulary. They evaluate retrieval and translation scores at different layers; gold alignments are easy to derive given that the corpora are effectively parallel corpora, and that the vocabularies for both halves are effectively the same. As we build on these experiments, we adopt slightly simplified notation, and denote vocabulary-shifted corpora with square brackets, eg. [EN].

\section{Experiments}

Galactic Dependencies A drawback of the multilingual experiments presented in Dufter and Schütze (2021) is that EN and [EN] effectively have the same structure. While the authors attempt to control for this in additional experiment where word order in [EN] is completely reversed, this does not resemble realistic differences across languages. Using true multilingual corpora is, however, difficult: our retrieval and translation tasks are easy to bootstrap precisely because we have faux-parallel corpora, with effectively pre-aligned vocabulary.

To induce structural diversity in our corpora, therefore, we reorder our corpora using Galactic Dependencies (GD) models (Wang and Eisner, 2016). Briefly, GD models sample ordering statistics based on dependency relations for the dependants of verbs and/or nouns from some superstrate language XX; when applied to sentences

these during fine-tuning helps cross-lingual zero-shot generalization. in some substrate language (in the context of our experiments, EN), the models reorder dependants of VERB and/or NOUN nodes to match the ordering statistics of the substrate language they were trained on. We opt to reorder both nominal and verbal arguments, and follow the authors in denoting the sampling operation with a $\sim$, giving us for eg. EN $\sim \mathrm{XX}$ for an English language corpus, with dependent order statistics adapted from some language XX. Table 2 contains an example sentence and some of its reorderings.

Note that GD reordering only works for projective sentences, and rather than retain un-reordered non-projective sentences, we exclude them from all our corpora.

\begin{tabular}{cl}
\hline $\mathrm{EN}$ & So there were fourteen generations from Abraham to David. \\
$\mathrm{EN} \sim \mathrm{AR}$ & . there were So generations fourteen from Abraham to David \\
$\mathrm{EN} \sim \mathrm{DE}$ & there were So from Abraham to David fourteen generations . \\
$\mathrm{EN} \sim \mathrm{EU}$ & there were So David to Abraham from generations fourteen . \\
$\mathrm{EN} \sim \mathrm{FI}$ & Abraham from David to fourteen generations there were So . \\
$\mathrm{EN} \sim \mathrm{FR}$ & fourteen generations from Abraham to David were there So . \\
$\mathrm{EN} \sim \mathrm{HI}$ & there So David to Abraham from fourteen generations were. \\
$\mathrm{EN} \sim \mathrm{SV}$ & there were So generations from Abraham to David fourteen . \\
\hline
\end{tabular}

Table 2: An example sentence from the easy-to-read Bible with its GD reorderings.

This approach, while simple and useful, does have several limitations. Predominantly, because our reordering is fundamentally syntactic/structural, our fake languages still maintain both the morphology of the source language (English in our case), and the same vocabulary distribution. Thus, although scrambling ought to affect context and neighbourhoods, an English token and its corresponding fake token have exactly the same unigram distribution.

Training Our model of choice is an underparameterised BERT, as in Dufter and Schütze (2021). We train multiple such underparameterised BERT models, each with a different encoding mechanism from Section 2, on two bilingual corpora:

EN + [EN] - a bilingual corpus comprised of English, and a fake vocab-shifted English.

$\mathbf{E N}+[\mathbf{E N} \sim \mathbf{X X}]-$ a bilingual corpus comprised of English, and a fake English that has had its constituents reordered to match the distribution of some language $\mathrm{XX}$.

We reorder our English starting point according to seven different faux-languages (just "languages" for brevity): Arabic, German, Basque, Finnish, 

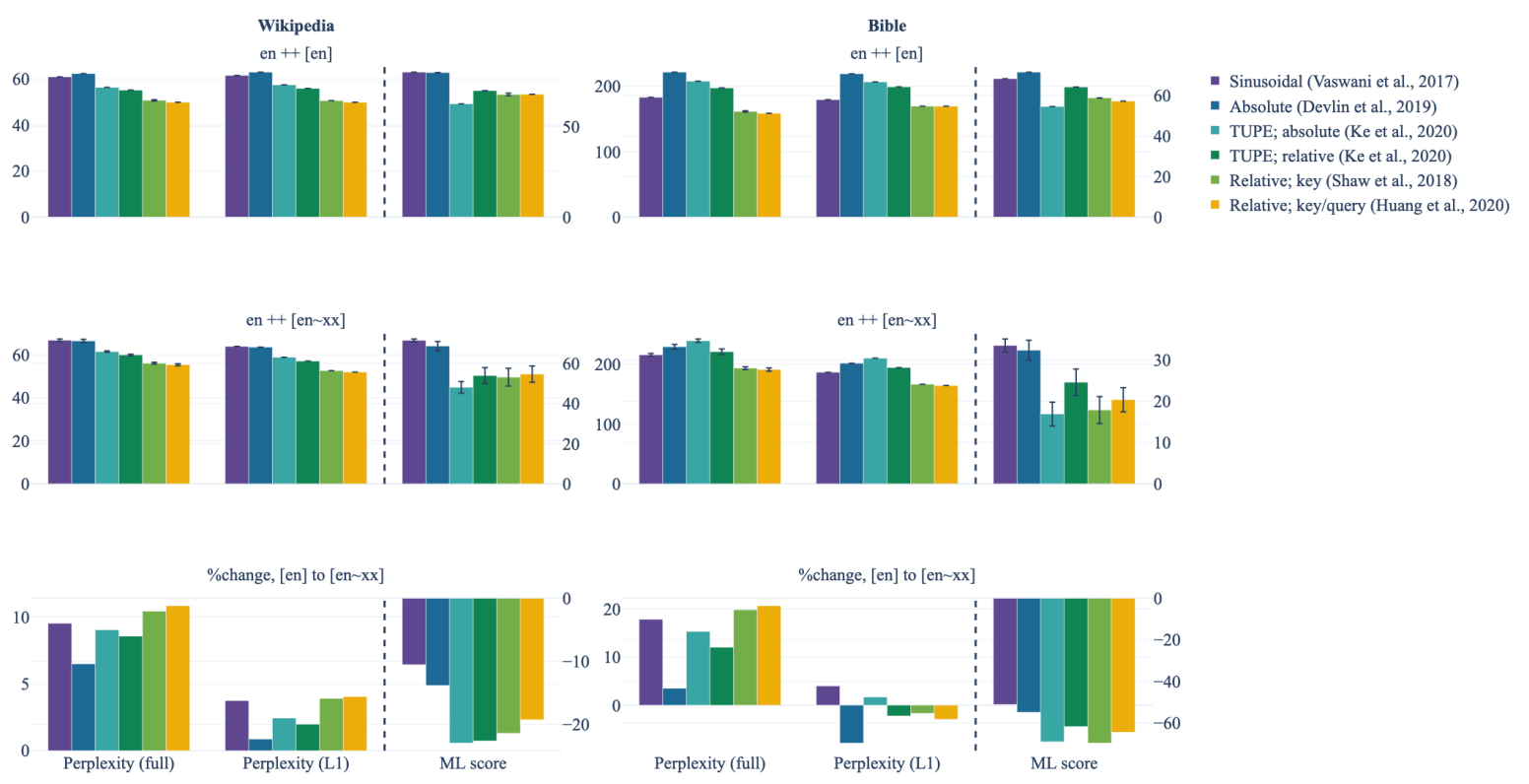

Figure 1: Main results: While untied and relative positional encodings are superior to sinusoidal and absolute positional encodings in the monolingual setting, they are clearly worse in the multilingual setting, especially for structurally different languages. The multilingual (ML) scores are computed as in Dufter and Schütze (2021). Note also that results are averages across seven different word orders (see $\S 3$ ).

French, Hindi and Swedish. Note that given that our starting point was English, there was no way for us to control for morphological differences; as such, languages with freer word order (like Basque) are likelier to make our English corpora ambiguous.

We use two corpora in this work: the first is the Bible splits from Dufter and Schütze (2021), with the English easy-to-read Bible as the training split, and the KJV Bible as validation. The second corpus uses the English Wikipedia as the training split, and Common Crawl as validation. We present corpus statistics in Table 3. For each corpus, we learn and apply a BPE vocabulary of size 2048 .

\begin{tabular}{ccc} 
& Train & Validation \\
\hline Bible & 30602 & 9080 \\
Wikipedia & 50000 & 20000 \\
\hline
\end{tabular}

Table 3: Corpus sizes in sentences (two languages per corpus)

Following Dufter and Schütze (2021), our BERT models all have a single head and 12 layers. We reduce the dimensionality of the encoder layers to 64, and the feed-forward layers to 256. Each model is trained for 100 epochs with three different random seeds $(0,42$ and 100), giving us a total of 7 languages x 6 encoding methods x 3 seeds $\mathrm{x} 2$ cor- pora $=252$ models. We implement our code ${ }^{3}$ in the transformers library (Wolf et al., 2020). For learned absolute and the two relative encoding models, we use the default implementations, that scale attention operations by a scaling factor of $\frac{1}{\sqrt{d}}$. For our untied models, we adjust our scaling factor to $\frac{1}{\sqrt{2 d}}$ as in the original paper (Ke et al., 2020). For sinusoidal representations, while Vaswani et al. (2017) multiply token embeddings by $\sqrt{d}$ to avoid drowning them out with the $[-1,1]$ sinusoidal encoding range, we find that our default embedding size is too small for this to have an effect, and instead scale up token embeddings by $2 \sqrt{d}$ before adding positional encodings.

For all parameterised encoding models except TUPE (relative), we use a maximum of $k=512$ positions; the concrete transformers implementation of the relative methods means that this gives us 1023 total offsets. ${ }^{4}$ For TUPE (relative), we use a maximum of $k=128$ positions, divided into 32 bins with logarithmically increasing bin sizes; this is taken from the original implementation in $\mathrm{Ke}$ et al. (2020).

\footnotetext{
${ }^{3}$ github.uio.no/vinitr/ multilingual-position

${ }^{4}$ In line with Shaw et al. (2018), we also attempted to use $k=16$ for the relative key model, but saw no difference in results.
} 


\section{Evaluation}

We adopt Dufter and Schütze's (2021) evaluation pipeline, evaluating each of our models at layers 0 and 8; we also describe a multilingual score, which is defined as the average accuracy for the retrieval and translation tasks, at layers 0 and 8 . We also measure perplexity, both on the monolingual first half of the corpus, and on both halves combined. Note that true perplexities for masked language models are intractable (Wang and Cho, 2019; Salazar et al., 2020). We use a trivial approximation and calculate perplexity based on the prediction loss for each masked token; note that while these suffice for comparison purposes, they are not true perplexities and should not be taken as such outside the context of these experiments.

We present our results (averaged out over fauxlanguages) in Figure 1, with full results in Appendix C. As expected, the more recent positional encodings are superior to sinusoidal or absolute positional encodings in the monolingual setting; but somewhat surprisingly, sinusoidal and absolute positional encodings are clearly outperforming the more recent approaches in the multilingual setting. We also note that the gap in multilingual performance only grows larger when a different word order is imposed on the target language; see the bottom row of Figure 1. Interestingly, switching to structurally different L2s can sometimes reduce the language modelling perplexity of the L1: this could be due to regularisation induced by structural differences.

Typological differences We discuss "typology" with a caveat: our experiments with GD only alter word order, which means that all our alteredstructure experiments still have English morphology. As such, it is impossible to talk about nonEnglish languages; only about non-English wordorder tendencies, when induced in English. Having said that, when we measure performance variation across languages (Figure 2), our results are more or less what one would expect: performance is decent for relatively rigid word-order languages, and poorer for languages that have complex morphology.

Interestingly, SVO languages consistently tend to perform better than our three non-SVO languages (Basque, Hindi and Arabic); this could be due to $\mathrm{VSO} / \mathrm{SOV}$ languages requiring morphology to disambiguate between adjacent nominals (Lev-

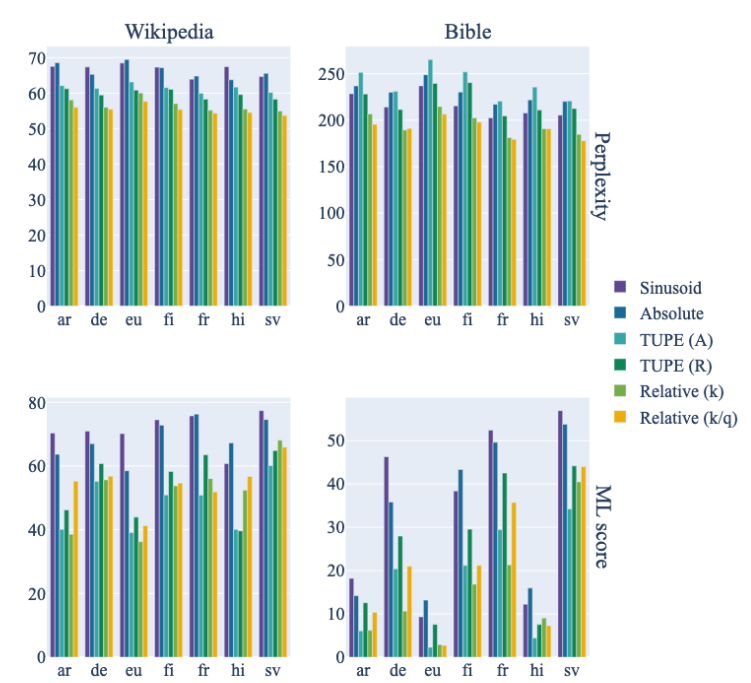

Figure 2: (Full) perplexity and ML score across languages.

shina, 2019). Another justification could also be that these are languages with a very different "default" word order to English; this would further motivate Ding et al.'s (2020) use of cross-lingually reordered position markers.

Real-world results While we conduct most of our analyses on our toy models, we also ran a series of experiments to verify that our results would hold with larger models. As such, we pre-trained full size BERT models (base, not large) for two epochs, on a corpus consisting of $8.5 \mathrm{M}, 9.3 \mathrm{M}$ and $800 \mathrm{k}$ sentences in English, German and Hindi respectively. We then fine-tuned these models for three epochs on (English) MultiNLI (Williams et al., 2018), and evaluated on held-out XNLI test sets for our three languages (Conneau et al., 2018); the process took approximately 4 days per model, on a single V100 GPU. We trained two models (seeds 0 and 42) per method, for three different positional encoding methods: a) absolute positional encodings, as these are used in the original BERT, b) sinusoidal encodings, as these were the original transformer encodings, and c) TUPE (absolute), as the most recent innovation. Our real-world results appear to validate our toy experiments: performance on English, the language the model was fine-tuned on, is highest with TUPE, while cross-lingual transfer suffers, both on German and to a lesser extent on Hindi. 


\begin{tabular}{|c|c|c|c|c|c|c|c|c|c|c|c|c|c|c|}
\hline \multirow{3}{*}{ Embedding } & \multicolumn{7}{|c|}{ Wiki/CC } & \multicolumn{7}{|c|}{ Bible } \\
\hline & \multicolumn{2}{|c|}{ Perplexity } & \multicolumn{2}{|c|}{ Retrieval } & \multicolumn{2}{|c|}{ Translation } & \multirow{2}{*}{ ML score } & \multicolumn{2}{|c|}{ Perplexity } & \multicolumn{2}{|c|}{ Retrieval } & \multicolumn{2}{|c|}{ Translation } & \multirow{2}{*}{ ML score } \\
\hline & Full & L1 & 0 & 8 & 0 & 8 & & Full & L1 & 0 & 8 & 0 & 8 & \\
\hline Sinusoidal & 66.73 & 63.96 & 37.43 & 97.29 & 77.03 & 64.07 & 68.95 & 215.77 & 186.53 & 4.82 & 54.09 & 47.09 & 27.62 & 33.4 \\
\hline Absolute & 66.35 & 63.44 & 52.35 & 96.53 & 76.05 & 53.62 & 68.59 & 229.28 & 201.88 & 9.62 & 52.51 & 47.6 & 19.36 & 32.27 \\
\hline TUPE (absolute) & 61.4 & 58.77 & 9.61 & 84.72 & 65.89 & 36.57 & 48.07 & 239.48 & 210.16 & 1.65 & 28.86 & 28.85 & 8.12 & 16.87 \\
\hline TUPE (relative) & 59.81 & 56.96 & 16.25 & 88.5 & 71.7 & 40.54 & 53.89 & 221.04 & 194.58 & 2.54 & 41.34 & 40.39 & 13.92 & 24.55 \\
\hline Relative (key) & 55.98 & 52.5 & 20.2 & 87.36 & 73.09 & 31.38 & 53.09 & 193.49 & 166.75 & 2.18 & 28.46 & 30.43 & 10.25 & 17.83 \\
\hline Relative (key/query) & 55.28 & 51.83 & 21.24 & 88.04 & 73.58 & 34.42 & 54.64 & 191.23 & 164.41 & 2.4 & 31.6 & 34.26 & 13.03 & 20.32 \\
\hline
\end{tabular}

Table 4: Detailed results, averaged across our faux-languages. Best results per metric in bold.

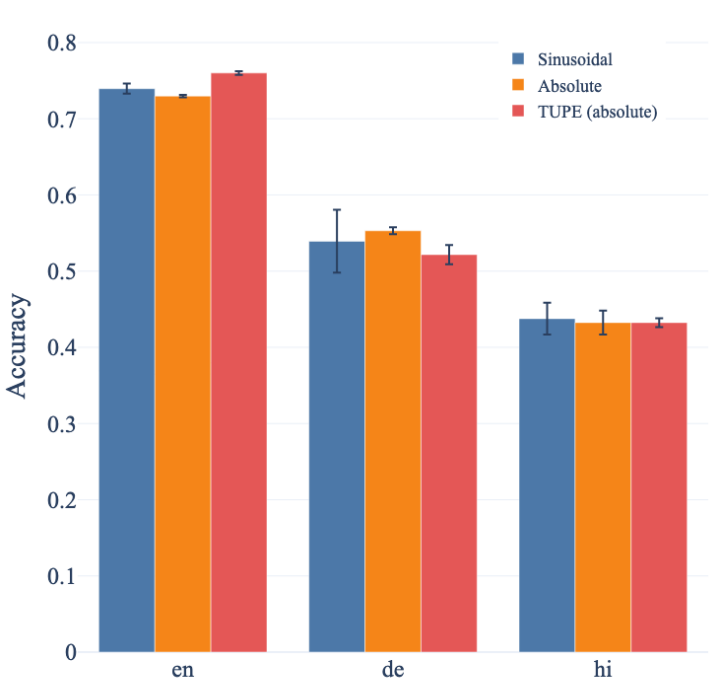

Figure 3: Real-world results on XNLI. Models were pretrained on a large text corpus and finetuned on English MultiNLI.

\section{Analyses}

In an attempt to explain the significantly improved cross-lingual performance of absolute positional encodings, we tried to examine precisely what sort of encoding was being learnt. Part of the original motivation behind sinusoidal encodings was that they would allow for compositionality; for any fixed offset $k$, there exists a linear transformation from $p_{p o s}$ to $p_{p o s+k}$, making it easier to learn to attend to relative offsets; the proof of this is in Appendix A. ${ }^{5}$

We examined our absolute positional encodings to see whether or not they were being induced to learn some specific function. Figure 4 plots 4 dimensions of absolute and TUPE(a) positional encoding, for the EN $+[\mathrm{EN} \sim \mathrm{FI}]$ model; each line

\footnotetext{
${ }^{5}$ Vaswani et al. (2017) do not explicitly mention compositionality, but only generalization across positions for fixed offsets. Positional disentanglement is the flipside of compositionality, however (Chaabouni et al., 2020).
}

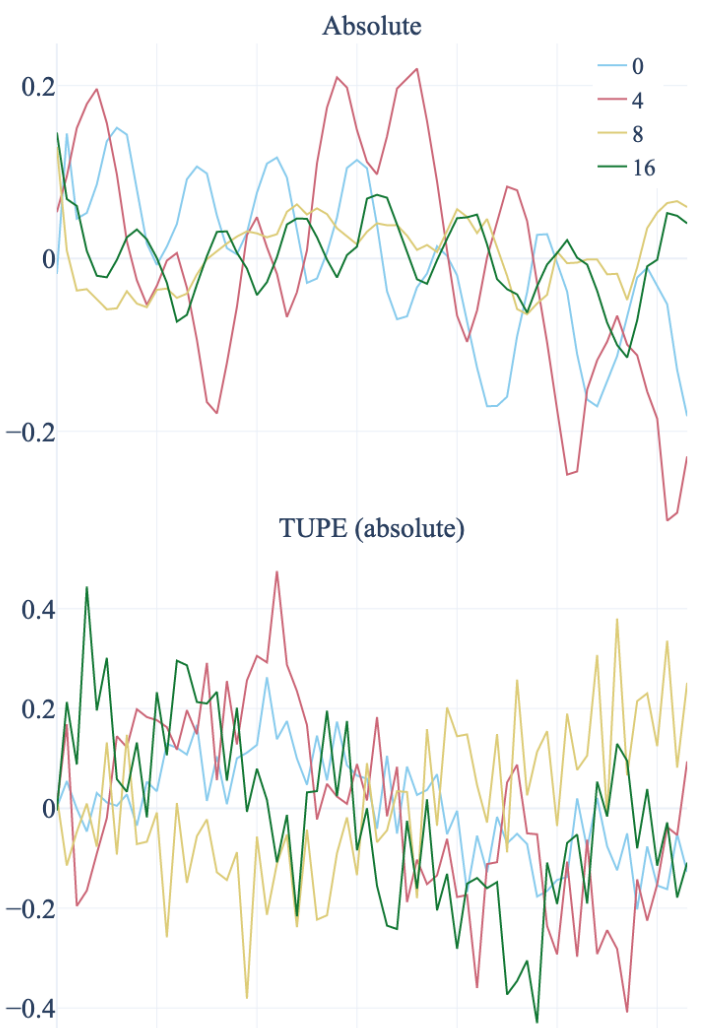

Figure 4: Dimensions 0, 4, 8 and 16 of learnt absolute and TUPE positional encodings over 32 positions for EN $\sim$ FI, seed 0 .

represents a specific dimension of the encoding vectors generated for positions 0 to 31 . Interestingly, it appears that absolute representations converge to waveforms that represent sinusoids somewhat, while neither of the untied experiments do so (cf. Appendix B).

We hypothesize that absolute representations converge to waveforms because of increased pressure for compositionality, being trained on structurally different languages. To test this, we quantify the extent to which the absolute, relative and untied encodings are compositional in the sense that there 


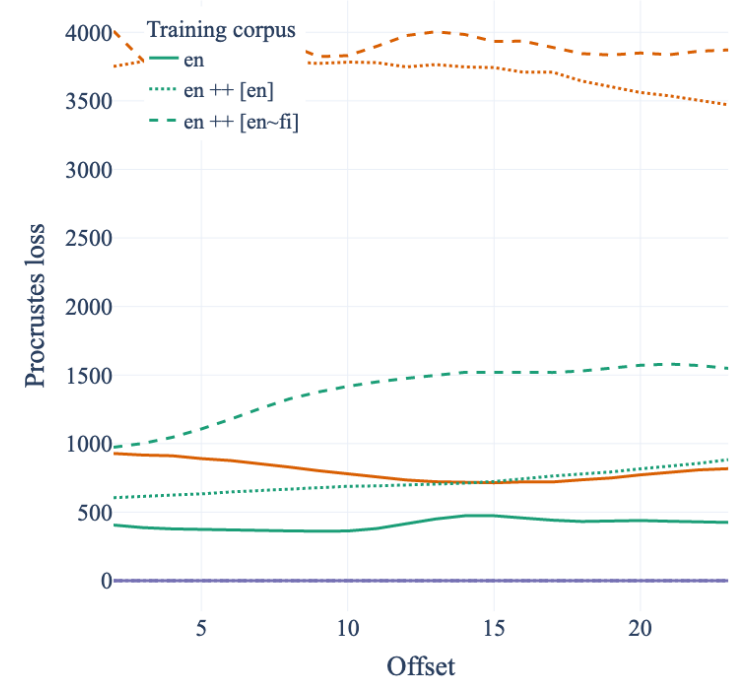

Figure 5: Procrustes loss for absolute encodings and TUPE (seed 0); differences are statistically significant with $p<0.001$ (Wilcoxon). The sinusoidal loss is $\approx 0$.

is a linear transformation from $p_{p o s}$ to $p_{p o s+k}$ for different $k$.

To this end, we use Procrustes analysis (Stegmann and Gomez, 2002) to learn a linear transformation for each $k$, based on the representations of $p_{\text {pos }}$ and $p_{\text {pos }+k}$. Specifically, we apply orthogonal Procrustes analyses (Schönemann, 1966), which avoid scaling and translation.

First, we minimise $\arg \min _{\mathrm{T}}\left\|p_{\text {pos }}-\mathrm{T} p_{\text {pos }+k}\right\|^{2}$. Next, we apply $\mathrm{T}$ to a different randomly selected pos ${ }^{\prime}$, i.e. we calculate $\mathcal{L}=\left\|p_{\text {pos }}-\mathrm{T} p_{\text {pos }}+k\right\|^{2}$. The higher the final loss $\mathcal{L}$, the less our encodings facilitate compositionality. In order to make learning $\mathrm{T}$ simpler, rather than selecting representations for single positions pos and $p^{\prime} s^{\prime}$, we select chunks of arbitrary size $C$, and stack their positions into a matrix. Note that for sinusoidal representations, the loss is close to zero regardless of span.

The losses are plotted over a range of offsets for both absolute representations and for TUPE(a), in Figure 5; we include a control model trained on a monolingual corpus. Losses are averaged over 125 runs per offset, with random values of pos, pos $^{\prime}$ and $C$. While both forms of representation appear to be similar (and relatively non-sinusoidal) when trained on the monolingual corpus, introducing bilingualism leads to a clear difference between the two: absolute positional representations tend to be a lot closer to sinusoidal representations than untied ones do. Note, also, that this gap is clear- est for the (simpler) EN $+[\mathrm{EN}]$ experiment - this is unsurprising, as $\mathrm{EN}+[\mathrm{EN}]$ is still perceived as bilingual due to the shifted vocabulary. The structural similarity between the two, however, makes it easier to build compositional representations by relying on offsets, as the model only needs to learn to represent one language, structurally speaking. We observe a similar gap when comparing pretrained BERT models: bert-base-multilingual-cased exhibits more sinusoidal representations over a range of offsets, when compared to bert-base-cased, although the gap is narrower than with our toy models.

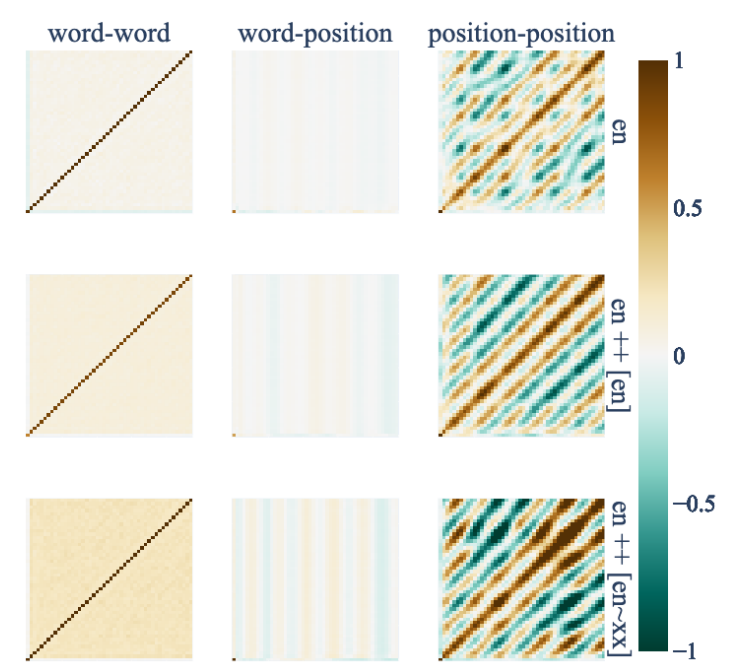

Figure 6: Word-position correlations for our Finnishreordered model with random seed 0 ; words on the yand positions on the $\mathrm{x}$-axis.

Correlations in multilingual settings A key motivation for eliminating word-position correlations, presented in (Ke et al., 2020), is the fact that these correlations are effectively zero, leading to no additional information for the model. Figure 6 captures word-position correlations from three of our trained models (with an additional model trained on a purely monolingual corpus); note that while these correlations are very close to zero for monolingual corpora, there is a visible "banding" phenomenon in the multilingual corpora, that only grows stronger when a different grammar is sampled. A similar banding phenomenon is visible when we compare multilingual and monolingual pre-trained BERT models (Appendix B), albeit with reduced magnitude. We hypothesize 


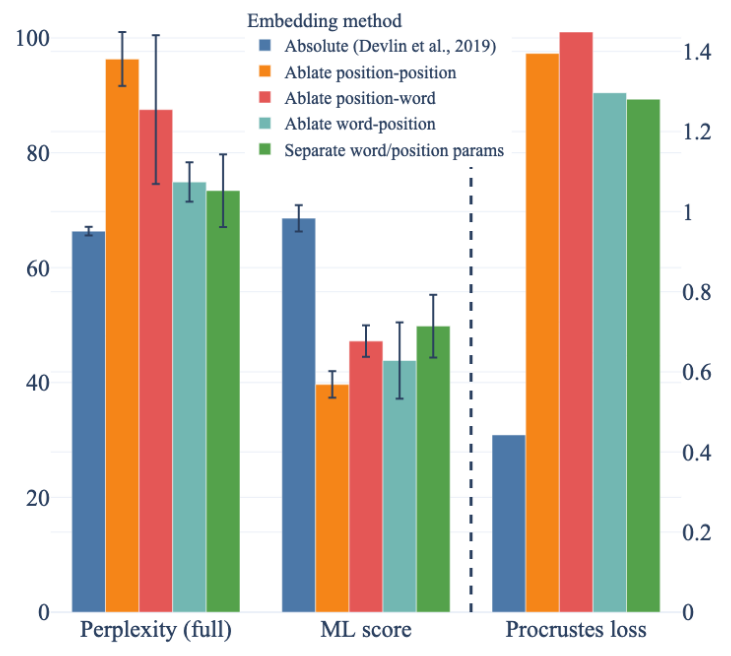

Figure 7: Ablation experiments, averaged over languages (for perplexity and ML score). Procrustes losses calculated as in $\$ 5$, for the $\mathrm{EN} \sim$ FI model (seed $0)$.

that the pressure for compositionality induces these correlations.

Ablation studies Finally, we ran a series of ablation experiments on absolute positional encodings to support the above analysis. Three of the experiments involved removing position-position correlations, position-word correlations, word-position correlations, and a fourth involved using separate parameters for word and position attention. Results are presented in Figure 7; we also include the median Procrustes loss. We note that the removal of both position-word correlations and word-position correlations has an effect on both perplexity and ML score. Interestingly, removing word-position correlations $\left(\left(p_{i} W^{Q}\right)\left(w_{j} W^{K}\right)^{\top}\right)$ does not have the same effect as the inverse does: perplexity is lower than with position-word correlations removed, but so is the ML score, indicating a difference between the role played by position as a key, and as a query.

On relative representations Given our previous assumptions about offsets aiding compositionality, why, then, do our relative representations - that explicitly calculate offsets - perform poorly in multilingual settings? We speculate that the reason relative encodings appear to hurt multilingual compression is that offset-specific bias terms sparsify the learning signal for (and thereby hinder the alignment of) disjoint vocabularies. In compensating for this, relative positional encodings sacrifice their compositionality. Relative representations aid compositionality by directly providing a bias term derived from the distance between a word pair. As shown above, absolute representations learn similar biases; however, being actively forced to learn such biases could encourage models to jointly learn alignment and compositionality.

Further, offset representations are also effectively "hard", i.e. derived from the hard distance between the two tokens. The interaction between $w_{i}$ and $w_{j}$ is not wholly mediated by the distance $i-j$, however, this correlation is forced by the product term $\left(x_{i} W^{Q}\right)\left(a_{i j}\right)^{\top}$. The term $\left(x_{i} W^{Q}\right)\left(p_{j} W^{K}\right)^{\top}$, on the other hand, could effectively attend to multiple offsets. $p_{j} W^{K}$ is fixed for position $j$; given the sinusoidal nature of $p$, the product term could induce a "soft" positional representation with subspaces attending to different offsets ${ }^{6}$; the relevant offset mix could then be indexed into by $x_{i} W^{Q}$.

\section{Discussion}

The main contribution of our work is practical, namely showing that findings about positional encodings in the context of monolingual language models do not apply straightforwardly to multilingual language models. In answering why sinusoidal embeddings are superior to more recent alternatives in the multilingual setting, we also found the compositionality of positional encodings to be predictive of multilingual compression in such models. While relative positional encodings seem designed for compositionality, they prevent efficient alignment of multilingual vocabularies.

Sinha et al. (2021) show that word order matters little for monolingual language model pretraining, and that pretrained language models seem to rely mostly on higher-order word co-occurrence statistics. Our work shows that this finding does not generalize to pretraining multilingual language models. In the multilingual setting, word order clearly matters, as also shown in previous work (Ke et al., 2020; Dufter and Schütze, 2021), and compositional positional encodings seem to facilitate effective multilingual compression. This aligns with the observation that syntactic reordering à la Ding et al. (2020) is in some cases an effective way to encourage compositional cross-lingual representations.

\footnotetext{
${ }^{6}$ Indeed, we find that $p_{j} W^{k}$ is less invariant to Procrustes transformation than $p_{j}$ is.
} 
In general, our results illustrate how methods developed for monolingual language models should not be blindly adopted when training multilingual models, which potentially require different architectures. Conversely, we would encourage future work on new positional encoding mechanisms for non-autoregressive models to also evaluate these mechanisms in multilingual settings.

\section{Conclusion}

Through a series of synthetic and real experiments with training multilingual language models, we showed that a) sinusoidal positional encodings perform better in multilingual settings than more recent alternatives (that have been shown to perform better in monolingual settings); b) this is likely because of an increased pressure for compositionality. We devised a method for quantifying the compositionality of positional encodings, and strengthened our results by also considering word-position correlations and ablation studies.

\section{Acknowledgements}

We would like to thank Lilja Øvrelid, Erik Velldal, Mostafa Abdou and Artur Kulmizev for their feedback and comments. We would also like to acknowledge the supercomputing services provided by the CSC IT Center for Science in Finland.

\section{References}

Mikel Artetxe, Sebastian Ruder, and Dani Yogatama. 2020. On the Cross-lingual Transferability of Monolingual Representations. In Proceedings of the 58th Annual Meeting of the Association for Computational Linguistics, pages 4623-4637, Online. Association for Computational Linguistics.

Rahma Chaabouni, Eugene Kharitonov, Diane Bouchacourt, Emmanuel Dupoux, and Marco Baroni. 2020. Compositionality and generalization in emergent languages. In Proceedings of the 58th Annual Meeting of the Association for Computational Linguistics, pages 4427-4442, Online. Association for Computational Linguistics.

Alexis Conneau, Guillaume Lample, Ruty Rinott, Adina Williams, Samuel R. Bowman, Holger Schwenk, and Veselin Stoyanov. 2018. XNLI: Evaluating Cross-lingual Sentence Representations. arXiv:1809.05053 [cs].

Jacob Devlin, Ming-Wei Chang, Kenton Lee, and Kristina Toutanova. 2019. BERT: Pre-training of Deep Bidirectional Transformers for Language Understanding. arXiv:1810.04805 [cs].
Liang Ding, Longyue Wang, and Dacheng Tao. 2020. Self-Attention with Cross-Lingual Position Representation. arXiv:2004.13310 [cs].

Philipp Dufter, Martin Schmitt, and Hinrich Schütze. 2021. Position Information in Transformers: An Overview. arXiv:2102.11090 [cs].

Philipp Dufter and Hinrich Schütze. 2021. Identifying Necessary Elements for BERT's Multilinguality. arXiv:2005.00396 [cs].

Pengcheng He, Xiaodong Liu, Jianfeng Gao, and Weizhu Chen. 2021. DeBERTa: Decodingenhanced BERT with Disentangled Attention. arXiv:2006.03654 [cs].

Zhiheng Huang, Davis Liang, Peng Xu, and Bing Xiang. 2020. Improve Transformer Models with Better Relative Position Embeddings. arXiv:2009.13658 [cs].

Karthikeyan K, Zihan Wang, Stephen Mayhew, and Dan Roth. 2020. Cross-Lingual Ability of Multilingual BERT: An Empirical Study. arXiv:1912.07840 [cs].

Guolin Ke, Di He, and Tie-Yan Liu. 2020. Rethinking Positional Encoding in Language Pre-training. In International Conference on Learning Representations.

Natalia Levshina. 2019. Token-based typology and word order entropy: A study based on Universal Dependencies. Linguistic Typology, 23(3):533-572.

Danni Liu, Jan Niehues, James Cross, Francisco Guzmán, and Xian Li. 2020a. Improving Zero-Shot Translation by Disentangling Positional Information. arXiv:2012.15127 [cs].

Zihan Liu, Genta Indra Winata, Samuel Cahyawijaya, Andrea Madotto, Zhaojiang Lin, and Pascale Fung. 2020b. On the Importance of Word Order Information in Cross-lingual Sequence Labeling. arXiv:2001.11164 [cs].

Telmo Pires, Eva Schlinger, and Dan Garrette. 2019. How Multilingual is Multilingual BERT? In Proceedings of the 57th Annual Meeting of the Association for Computational Linguistics, pages 49965001, Florence, Italy. Association for Computational Linguistics.

Julian Salazar, Davis Liang, Toan Q. Nguyen, and Katrin Kirchhoff. 2020. Masked language model scoring. Proceedings of the 58th Annual Meeting of the Association for Computational Linguistics.

Peter H Schönemann. 1966. A generalized solution of the orthogonal procrustes problem. Psychometrika, 31(1):1-10.

Peter Shaw, Jakob Uszkoreit, and Ashish Vaswani. 2018. Self-Attention with Relative Position Representations. arXiv:1803.02155 [cs]. 
Koustuv Sinha, Robin Jia, Dieuwke Hupkes, Joelle Pineau, Adina Williams, and Douwe Kiela. 2021. Masked Language Modeling and the Distributional Hypothesis: Order Word Matters Pre-training for Little. arXiv:2104.06644 [cs].

Mikkel B. Stegmann and David Delgado Gomez. 2002. A brief introduction to statistical shape analysis. Informatics and Mathematical Modelling, Technical University of Denmark, DTU.

Ashish Vaswani, Noam Shazeer, Niki Parmar, Jakob Uszkoreit, Llion Jones, Aidan N. Gomez, Lukasz Kaiser, and Illia Polosukhin. 2017. Attention Is All You Need. arXiv:1706.03762 [cs]. ArXiv: 1706.03762 .

Alex Wang and Kyunghyun Cho. 2019. Bert has a mouth, and it must speak: Bert as a markov random field language model.

Benyou Wang, Lifeng Shang, Christina Lioma, Xin Jiang, Hao Yang, Qun Liu, and Jakob Grue Simonsen. 2021. ON POSITION EMBEDDINGS IN BERT. page 21.

Dingquan Wang and Jason Eisner. 2016. The Galactic Dependencies Treebanks: Getting More Data by Synthesizing New Languages. Transactions of the Association for Computational Linguistics, 4:491505.

Xing Wang, Zhaopeng Tu, Longyue Wang, and Shuming Shi. 2019. Self-Attention with Structural Position Representations. arXiv:1909.00383 [cs].

Adina Williams, Nikita Nangia, and Samuel Bowman. 2018. A Broad-Coverage Challenge Corpus for Sentence Understanding through Inference. In Proceedings of the 2018 Conference of the North American Chapter of the Association for Computational Linguistics: Human Language Technologies, Volume 1 (Long Papers), pages 1112-1122, New Orleans, Louisiana. Association for Computational Linguistics.

Thomas Wolf, Lysandre Debut, Victor Sanh, Julien Chaumond, Clement Delangue, Anthony Moi, Pierric Cistac, Tim Rault, Rémi Louf, Morgan Funtowicz, Joe Davison, Sam Shleifer, Patrick von Platen, Clara Ma, Yacine Jernite, Julien Plu, Canwen Xu, Teven Le Scao, Sylvain Gugger, Mariama Drame, Quentin Lhoest, and Alexander M. Rush. 2020. Transformers: State-of-the-art natural language processing. In Proceedings of the 2020 Conference on Empirical Methods in Natural Language Processing: System Demonstrations, pages 38-45, Online. Association for Computational Linguistics.

Shijie Wu and Mark Dredze. 2019. Beto, Bentz, Becas: The Surprising Cross-Lingual Effectiveness of BERT. In Proceedings of the 2019 Conference on Empirical Methods in Natural Language Processing and the 9th International Joint Conference on Natural Language Processing (EMNLP-IJCNLP), pages
833-844, Hong Kong, China. Association for Computational Linguistics. 


\section{A Proof of linear transformability}

Let

$$
\left[\begin{array}{l}
\sin (\omega t) \\
\cos (\omega t)
\end{array}\right]
$$

represent a sine/cosine pair, characterised by position $t$. Let

$$
\mathrm{R}_{k}=\left[\begin{array}{cc}
\cos (\omega k) & \sin (\omega k) \\
-\sin (\omega k) & \cos (\omega k)
\end{array}\right]
$$

be a rotation matrix for angle $\omega k$. We then have:

$$
\begin{aligned}
R\left[\begin{array}{l}
\sin (\omega t) \\
\cos (\omega t)
\end{array}\right] & =\left[\begin{array}{cc}
\cos (\omega k) & \sin (\omega k) \\
-\sin (\omega k) & \cos (\omega k)
\end{array}\right] \cdot\left[\begin{array}{l}
\sin (\omega t) \\
\cos (\omega t)
\end{array}\right] \\
& =\left[\begin{array}{l}
\sin (\omega k) \cos (\omega t)+\cos (\omega k) \sin (\omega t) \\
\cos (\omega k) \cos (\omega t)-\sin (\omega k) \sin \omega t)
\end{array}\right] \\
& =\left[\begin{array}{l}
\sin (\omega(t+k)) \\
\cos (\omega(t+k))
\end{array}\right]
\end{aligned}
$$

implying that for a fixed frequency $\omega$, there exists a rotation matrix $\mathrm{R}_{k}$ that can induce a rotational offset of $k$.

\section{B Additional plots}




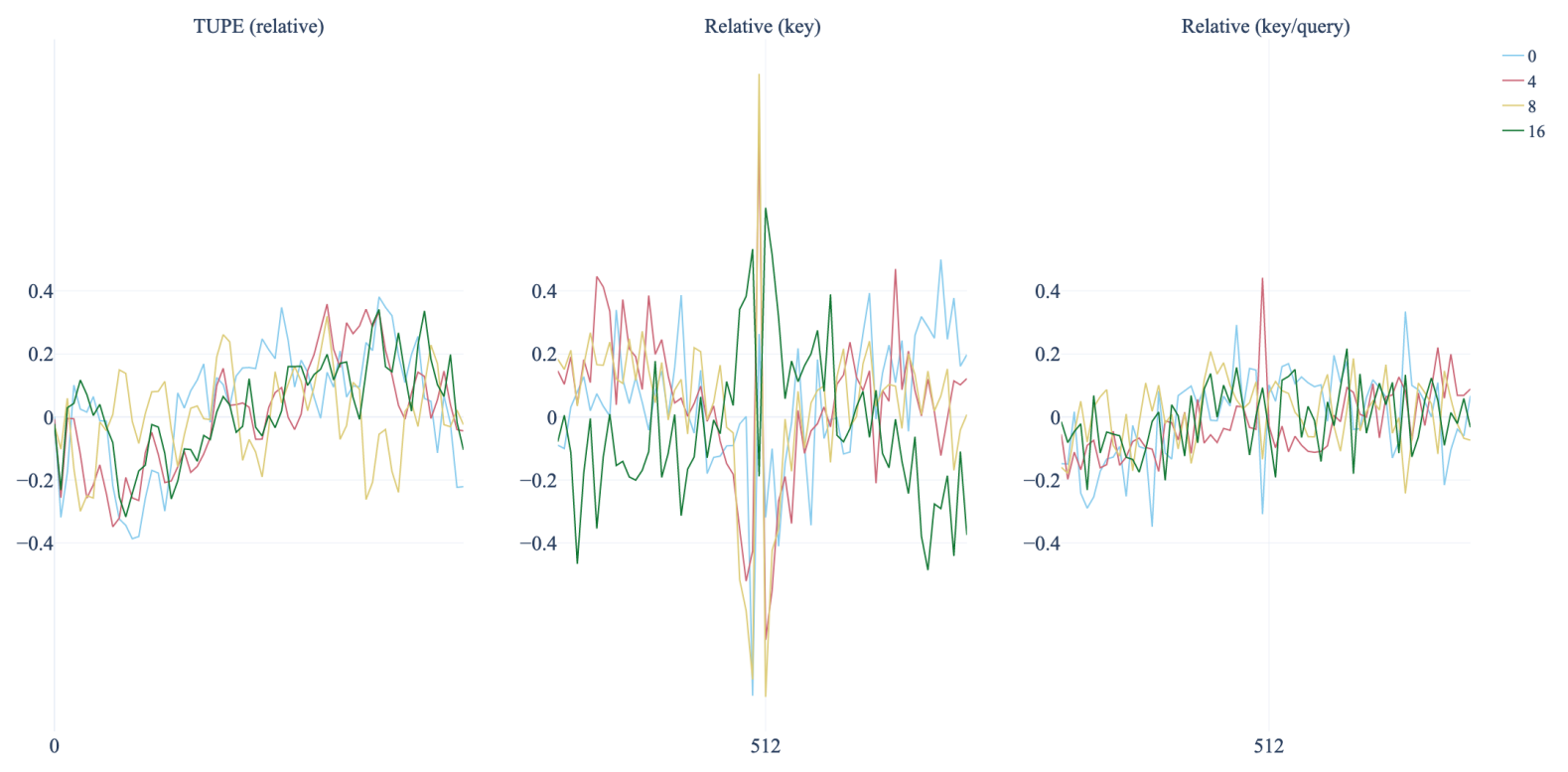

Figure 8: Four neurons over 32 for TUPE (relative); the same neurons for 32 offsets centred on 512 for the other relative models.

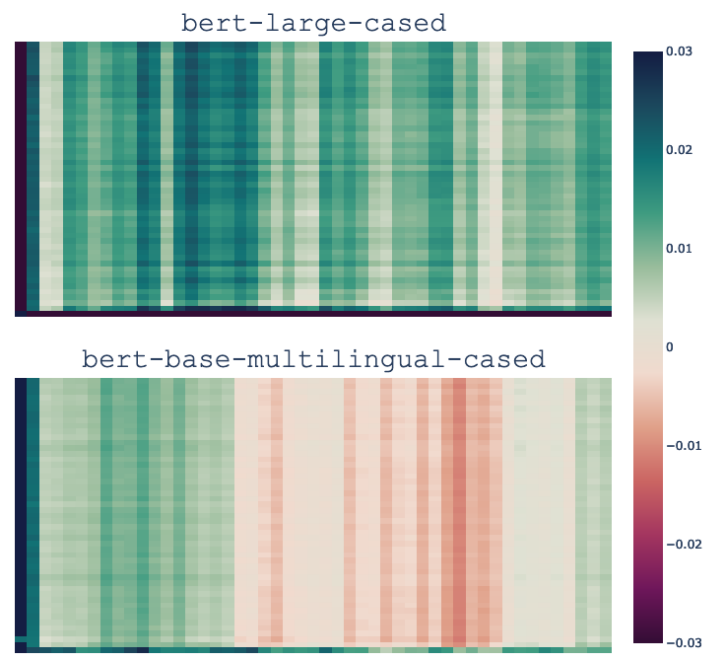

Figure 9: Word-position correlations for pretrained BERT models 
C Full results 


\begin{tabular}{|c|c|c|c|c|c|c|c|c|}
\hline \multirow{2}{*}{ Wiki/CC } & \multirow{2}{*}{ Embedding } & \multicolumn{2}{|c|}{ Perplexity } & \multicolumn{2}{|c|}{ Retrieval } & \multicolumn{2}{|c|}{ Translation } & \multirow{2}{*}{ ML score } \\
\hline & & Full & L1 & 0 & 8 & 0 & 8 & \\
\hline \multirow{6}{*}{ Arabic } & Sinusoidal & $67.52(1.72)$ & $64.16(2.14)$ & $47.90(25.39)$ & $98.09(0.90)$ & 71.75 (7.39) & $62.52(0.09)$ & $70.36(5.73)$ \\
\hline & Absolute & $68.54(2.01)$ & $65.02(2.83)$ & $44.84(8.00)$ & $95.14(0.98)$ & $70.79(3.62)$ & $46.74(10.01)$ & $63.67(3.37)$ \\
\hline & TUPE (a) & $62.09(0.57)$ & $59.01(0.45)$ & 7.88 (1.63) & $75.17(7.47)$ & $58.06(4.65)$ & $32.13(3.28)$ & $40.15(4.45)$ \\
\hline & TUPE (r) & $61.22(0.50)$ & $57.81(1.05)$ & $15.12(4.39)$ & $83.44(13.58)$ & $64.94(9.55)$ & $29.03(10.33)$ & 46.22 (10.09) \\
\hline & Relative (k) & $58.08(0.98)$ & $53.92(0.89)$ & $12.61(5.00)$ & $74.14(15.52)$ & $63.27(13.04)$ & $9.37(3.85)$ & $38.56(9.24)$ \\
\hline & Relative (k/q) & $55.96(1.46)$ & $51.98(1.02)$ & $20.95(8.34)$ & 89.53 (8.39) & $73.01(5.47)$ & $35.83(20.20)$ & $55.23(12.46)$ \\
\hline \multirow{6}{*}{ German } & Sinusoidal & $67.34(1.52)$ & $64.74(1.21)$ & $47.12(25.19)$ & $98.94(0.22)$ & $71.53(14.37)$ & $61.06(10.25)$ & 70.95 (3.74) \\
\hline & Absolute & 65.28 (1.69) & $62.67(2.08)$ & $63.68(2.44)$ & $98.87(0.06)$ & $80.21(0.60)$ & $36.34(24.86)$ & $67.01(8.44)$ \\
\hline & TUPE (a) & $61.29(0.70)$ & $58.70(0.60)$ & $12.11(1.29)$ & $92.87(2.50)$ & $71.39(3.10)$ & $48.28(3.36)$ & 55.15 (2.09) \\
\hline & TUPE (r) & $59.42(0.77)$ & $56.90(1.29)$ & $18.27(3.33)$ & $97.40(1.13)$ & $77.86(2.10)$ & $43.72(13.84)$ & $60.74(5.49)$ \\
\hline & Relative (k) & $56.00(0.22)$ & $52.29(1.23)$ & $23.00(5.06)$ & $95.70(3.57)$ & 79.66 (1.94) & $26.08(17.72)$ & $55.67(8.05)$ \\
\hline & Relative (k/q) & $55.45(0.73)$ & 51.77 (1.49) & $22.67(7.54)$ & $90.80(10.64)$ & $76.50(7.72)$ & $39.51(18.52)$ & 56.83 (11.69) \\
\hline \multirow{6}{*}{ Basque } & Sinusoidal & $68.44(0.88)$ & $64.15(1.30)$ & $48.65(24.10)$ & $97.01(0.70)$ & $70.63(7.73)$ & $61.65(2.26)$ & $70.16(5.46)$ \\
\hline & Absolute & $69.46(3.29)$ & $65.36(2.70)$ & $45.42(5.55)$ & $91.64(3.68)$ & $69.19(3.87)$ & $36.91(6.75)$ & $58.51(3.31)$ \\
\hline & TUPE (a) & $63.11(0.36)$ & $59.23(1.35)$ & $6.38(2.17)$ & 71.92 (12.96) & $57.18(7.90)$ & $28.21(16.17)$ & $39.13(10.48)$ \\
\hline & TUPE (r) & $60.82(0.86)$ & $56.61(1.77)$ & $10.85(2.47)$ & $77.57(12.22)$ & $64.37(6.83)$ & $33.41(12.54)$ & $43.95(8.01)$ \\
\hline & Relative (k) & $60.03(0.57)$ & $54.70(0.53)$ & $9.49(4.37)$ & $61.06(20.25)$ & $56.12(14.21)$ & $21.28(15.21)$ & 36.27 (12.29) \\
\hline & Relative (k/q) & $57.66(0.96)$ & $52.51(1.41)$ & $11.74(6.91)$ & $63.13(32.58)$ & $55.84(22.94)$ & $33.70(18.55)$ & 41.27 (20.79) \\
\hline \multirow{6}{*}{ Finnish } & Sinusoidal & $67.28(1.54)$ & $64.20(1.88)$ & $51.99(23.78)$ & $98.90(0.25)$ & $76.33(5.69)$ & $69.72(1.44)$ & $74.51(5.15)$ \\
\hline & Absolute & $67.14(2.20)$ & $64.04(2.76)$ & $51.91(11.81)$ & $97.90(1.13)$ & $77.54(0.67)$ & $63.53(7.04)$ & $72.83(1.87)$ \\
\hline & TUPE (a) & $61.53(1.21)$ & $58.06(0.95)$ & $11.45(1.36)$ & 91.69 (4.67) & $71.60(3.69)$ & $34.34(16.61)$ & $50.91(6.36)$ \\
\hline & TUPE (r) & $61.07(1.22)$ & $57.54(1.07)$ & $15.90(2.15)$ & $94.00(3.82)$ & $75.84(4.08)$ & $47.95(20.72)$ & $58.29(7.04)$ \\
\hline & Relative (k) & $57.05(1.14)$ & $53.13(0.43)$ & $22.31(3.20)$ & $96.01(0.70)$ & $78.96(0.68)$ & $20.75(7.37)$ & $53.76(0.78)$ \\
\hline & Relative (k/q) & $55.37(0.66)$ & $51.29(0.27)$ & $22.97(0.86)$ & $91.87(5.67)$ & $79.77(0.97)$ & $22.81(25.52)$ & $54.63(9.17)$ \\
\hline \multirow{6}{*}{ French } & Sinusoidal & $63.92(0.41)$ & $61.82(0.87)$ & $54.10(22.52)$ & $99.36(0.13)$ & $77.65(4.95)$ & $70.28(1.22)$ & $75.76(4.25)$ \\
\hline & Absolute & $64.76(1.10)$ & $62.68(1.37)$ & $59.12(13.35)$ & $99.14(0.37)$ & $79.57(0.63)$ & $68.90(2.75)$ & 76.28 (1.93) \\
\hline & TUPE (a) & $59.94(0.79)$ & $58.60(0.71)$ & $8.67(2.15)$ & $90.25(7.76)$ & $67.86(7.47)$ & 36.15 (7.99) & $50.87(6.57)$ \\
\hline & TUPE (r) & $58.28(0.78)$ & $56.14(1.37)$ & $19.36(3.62)$ & $96.64(2.93)$ & $78.50(1.72)$ & $51.63(18.73)$ & $63.50(7.99)$ \\
\hline & Relative (k) & $55.18(0.25)$ & $52.96(0.44)$ & $18.84(2.14)$ & $91.12(8.32)$ & $73.30(7.86)$ & $37.50(17.77)$ & $56.07(8.40)$ \\
\hline & Relative (k/q) & $54.35(0.23)$ & $52.10(1.02)$ & $19.01(5.15)$ & $90.09(9.29)$ & $72.22(7.48)$ & $26.51(22.43)$ & $51.88(10.57)$ \\
\hline \multirow{6}{*}{ Hindi } & Sinusoidal & $67.46(0.27)$ & $65.01(0.32)$ & $37.94(20.74)$ & $88.30(4.64)$ & $63.26(13.46)$ & $47.88(6.96)$ & $60.76(4.85)$ \\
\hline & Absolute & $63.75(1.05)$ & $61.38(0.97)$ & $47.98(0.66)$ & $95.58(2.19)$ & 76.18 (1.95) & $56.57(12.48)$ & $67.28(4.54)$ \\
\hline & TUPE (a) & $61.70(1.10)$ & $59.57(0.48)$ & $6.34(1.56)$ & $74.79(20.15)$ & $58.50(12.29)$ & $28.73(6.46)$ & $40.11(9.49)$ \\
\hline & TUPE (r) & $59.57(1.24)$ & $57.64(1.75)$ & $12.67(6.57)$ & $72.64(24.01)$ & $61.35(17.78)$ & $23.49(10.80)$ & 39.66 (14.67) \\
\hline & Relative (k) & $55.50(0.60)$ & $52.81(0.87)$ & $19.72(4.83)$ & $90.11(9.87)$ & $74.06(4.06)$ & $26.71(13.65)$ & $52.39(9.33)$ \\
\hline & Relative (k/q) & $54.51(0.21)$ & $52.04(0.74)$ & $23.45(6.88)$ & $93.27(6.35)$ & $75.60(3.41)$ & $32.95(21.46)$ & $56.72(10.94)$ \\
\hline \multirow{6}{*}{ Swedish } & Sinusoidal & $64.62(0.86)$ & $62.32(0.73)$ & $57.11(21.45)$ & $99.32(0.24)$ & $79.89(2.06)$ & $71.82(7.63)$ & $77.39(5.05)$ \\
\hline & Absolute & $65.54(1.86)$ & $62.95(2.48)$ & $53.52(4.43)$ & $97.45(0.79)$ & 78.85 (1.69) & $66.33(3.09)$ & $74.58(2.28)$ \\
\hline & TUPE (a) & $60.16(0.99)$ & $58.23(1.49)$ & $14.47(3.45)$ & $96.36(2.49)$ & $76.62(3.05)$ & $48.12(19.76)$ & $60.16(7.98)$ \\
\hline & TUPE (r) & $58.29(0.17)$ & $56.12(0.72)$ & $21.57(3.06)$ & $97.80(1.40)$ & 79.05 (3.18) & $54.56(2.06)$ & $64.86(1.83)$ \\
\hline & Relative (k) & $54.94(0.61)$ & $52.28(0.68)$ & $28.17(3.30)$ & $98.52(0.68)$ & $82.81(1.08)$ & $56.60(9.17)$ & $68.09(5.04)$ \\
\hline & Relative (k/q) & $53.66(0.22)$ & $51.16(1.11)$ & $27.87(4.86)$ & $97.63(2.04)$ & $82.11(2.22)$ & $49.65(17.66)$ & $65.89(8.57)$ \\
\hline
\end{tabular}




\begin{tabular}{|c|c|c|c|c|c|c|c|c|}
\hline \multirow{2}{*}{ Bible } & \multirow{2}{*}{ Embedding } & \multicolumn{2}{|c|}{ Perplexity } & \multicolumn{2}{|c|}{ Retrieval } & \multicolumn{2}{|c|}{ Translation } & \multirow{2}{*}{ ML score } \\
\hline & & Full & L1 & 0 & 8 & 0 & 8 & \\
\hline \multirow{6}{*}{ Arabic } & Sinusoidal & 228.44 (5.29) & $193.76(3.88)$ & $2.30(0.35)$ & $32.79(4.29)$ & $24.54(5.24)$ & $13.20(3.62)$ & $18.21(3.09)$ \\
\hline & Absolute & $236.83(8.40)$ & $206.44(10.04)$ & $4.18(0.20)$ & $22.72(5.92)$ & $24.18(3.04)$ & $5.75(1.87)$ & $14.21(2.67)$ \\
\hline & TUPE (a) & $251.32(5.55)$ & $209.54(9.00)$ & $0.72(0.36)$ & $10.18(6.12)$ & $9.91(5.73)$ & $3.44(2.47)$ & $6.07(3.67)$ \\
\hline & TUPE (r) & $228.09(18.36)$ & 193.67 (7.37) & $1.30(0.22)$ & $21.49(6.68)$ & $23.25(3.57)$ & $4.19(1.11)$ & $12.56(2.28)$ \\
\hline & Relative (k) & 206.77 (4.36) & $177.43(3.93)$ & $0.84(0.08)$ & $8.79(1.82)$ & $11.85(1.24)$ & $3.24(0.64)$ & $6.18(0.73)$ \\
\hline & Relative (k/q) & $195.47(5.84)$ & $166.20(2.53)$ & $1.44(0.60)$ & $15.87(8.02)$ & $19.30(7.44)$ & $4.82(2.64)$ & $10.36(4.52)$ \\
\hline \multirow{6}{*}{ German } & Sinusoidal & $214.19(8.29)$ & $185.11(3.92)$ & $6.80(1.86)$ & $76.04(3.25)$ & $64.60(2.83)$ & $37.76(5.90)$ & $46.30(2.53)$ \\
\hline & Absolute & $230.05(7.75)$ & $205.46(12.23)$ & $9.81(2.28)$ & $63.69(12.37)$ & $51.91(5.98)$ & $17.89(5.89)$ & $35.83(6.55)$ \\
\hline & TUPE (a) & $230.92(6.27)$ & $203.84(2.26)$ & $1.75(0.39)$ & $33.91(2.86)$ & $36.33(6.21)$ & $9.59(2.96)$ & $20.40(2.65)$ \\
\hline & TUPE (r) & $211.44(6.19)$ & $196.39(7.09)$ & $2.52(0.56)$ & $48.23(20.03)$ & $46.61(14.36)$ & $14.43(6.78)$ & $27.95(9.95)$ \\
\hline & Relative (k) & $189.45(4.10)$ & $165.36(2.59)$ & $1.50(0.48)$ & $16.44(7.67)$ & $19.43(8.06)$ & $5.12(2.08)$ & $10.62(4.56)$ \\
\hline & Relative (k/q) & $191.06(0.27)$ & $168.70(6.74)$ & $2.33(0.67)$ & $33.21(15.24)$ & $36.76(13.08)$ & $11.69(6.65)$ & $21.00(8.84)$ \\
\hline \multirow{6}{*}{ Basque } & Sinusoidal & $236.91(9.77)$ & $197.71(11.45)$ & $1.47(0.37)$ & $15.82(2.59)$ & $13.57(3.44)$ & $6.47(2.72)$ & $9.33(2.27)$ \\
\hline & Absolute & $248.87(18.53)$ & $212.26(14.12)$ & $4.90(0.82)$ & $24.07(4.75)$ & $17.72(2.05)$ & $6.00(2.44)$ & $13.17(1.15)$ \\
\hline & TUPE (a) & $265.28(11.20)$ & $220.59(17.75)$ & $0.42(0.18)$ & $2.98(1.30)$ & 4.57 (1.17) & $1.21(0.57)$ & $2.29(0.79)$ \\
\hline & TUPE (r) & $239.52(8.88)$ & $196.16(12.43)$ & $1.04(0.21)$ & $11.23(1.54)$ & $14.64(4.25)$ & $3.29(0.98)$ & $7.55(1.36)$ \\
\hline & Relative (k) & $214.59(5.79)$ & $170.40(2.68)$ & $0.52(0.14)$ & $3.24(1.20)$ & $6.23(1.05)$ & $1.60(0.67)$ & $2.90(0.62)$ \\
\hline & Relative (k/q) & $206.33(6.43)$ & $166.16(2.67)$ & $0.49(0.18)$ & $3.15(1.43)$ & $5.96(2.18)$ & $1.37(0.86)$ & $2.74(1.09)$ \\
\hline \multirow{6}{*}{ Finnish } & Sinusoidal & $215.28(2.80)$ & $181.92(5.08)$ & $4.34(0.29)$ & $64.24(9.50)$ & $56.92(5.55)$ & $28.02(8.05)$ & $38.38(5.73)$ \\
\hline & Absolute & $230.22(12.83)$ & $194.61(4.17)$ & $14.40(3.69)$ & $68.82(5.06)$ & $63.92(5.37)$ & $26.18(8.15)$ & $43.33(5.03)$ \\
\hline & TUPE (a) & $251.91(4.75)$ & $215.41(10.58)$ & $1.94(0.56)$ & $35.29(11.75)$ & $36.96(8.56)$ & $10.58(6.28)$ & $21.19(6.73)$ \\
\hline & TUPE (r) & $240.35(7.43)$ & 206.09 (14.62) & $3.00(0.40)$ & $52.44(7.90)$ & $53.54(5.29)$ & $9.22(5.12)$ & $29.55(3.15)$ \\
\hline & Relative (k) & $202.40(8.09)$ & $170.90(9.54)$ & $1.77(0.90)$ & $23.66(13.30)$ & 30.67 (14.17) & $11.30(5.45)$ & $16.85(8.23)$ \\
\hline & Relative (k/q) & $197.92(5.13)$ & $161.33(8.55)$ & $2.38(0.70)$ & $29.05(3.31)$ & $40.63(10.06)$ & $12.74(5.02)$ & $21.20(4.70)$ \\
\hline \multirow{6}{*}{ French } & Sinusoidal & 202.44 (4.97) & $179.19(6.13)$ & $7.36(1.66)$ & $82.96(1.61)$ & $74.46(1.00)$ & $44.95(4.13)$ & $52.43(0.76)$ \\
\hline & Absolute & $217.10(11.25)$ & $199.77(10.51)$ & $13.60(1.64)$ & $75.18(7.83)$ & $74.33(5.80)$ & 35.35 (9.98) & $49.62(6.21)$ \\
\hline & TUPE (a) & $220.48(9.85)$ & $203.21(12.12)$ & $2.49(0.35)$ & $52.95(15.51)$ & $46.47(10.33)$ & $15.98(5.82)$ & $29.47(7.80)$ \\
\hline & TUPE (r) & $204.57(8.86)$ & $188.30(14.18)$ & $4.07(0.72)$ & $71.33(10.07)$ & $65.27(6.85)$ & $29.41(8.71)$ & $42.52(6.50)$ \\
\hline & Relative (k) & $181.31(2.67)$ & $161.53(3.44)$ & $2.36(1.09)$ & $36.83(20.95)$ & $33.84(15.39)$ & $12.17(9.58)$ & $21.30(10.92)$ \\
\hline & Relative (k/q) & $179.43(9.50)$ & $159.12(8.66)$ & $4.01(0.93)$ & $57.36(10.80)$ & $56.62(9.44)$ & $24.81(10.53)$ & $35.70(6.91)$ \\
\hline \multirow{6}{*}{ Hindi } & Sinusoidal & $207.71(3.54)$ & $187.60(4.39)$ & $2.08(0.53)$ & $22.03(8.05)$ & $17.30(5.85)$ & $7.54(2.13)$ & $12.24(4.13)$ \\
\hline & Absolute & $221.81(6.29)$ & $197.47(9.65)$ & $4.62(1.64)$ & $29.81(13.95)$ & $23.44(9.49)$ & $6.00(1.86)$ & $15.97(6.55)$ \\
\hline & TUPE (a) & 235.64 (11.94) & $216.64(15.13)$ & $0.70(0.06)$ & $5.63(1.00)$ & $9.15(2.07)$ & $2.31(0.57)$ & $4.45(0.69)$ \\
\hline & TUPE (r) & $210.78(8.59)$ & $189.10(9.12)$ & $0.83(0.37)$ & $12.64(5.58)$ & $13.41(6.40)$ & $3.26(1.76)$ & $7.54(3.52)$ \\
\hline & Relative (k) & $190.88(4.72)$ & $172.32(7.12)$ & $1.24(0.43)$ & $15.41(6.60)$ & $14.80(6.91)$ & $4.71(2.33)$ & $9.04(4.06)$ \\
\hline & Relative (k/q) & $190.68(6.60)$ & $168.92(4.95)$ & $1.06(0.32)$ & $11.42(5.32)$ & $13.01(4.36)$ & $3.59(0.56)$ & $7.27(2.62)$ \\
\hline \multirow{6}{*}{ Swedish } & Sinusoidal & $205.42(4.67)$ & $180.45(2.27)$ & $9.41(0.30)$ & $84.72(2.59)$ & $78.21(0.52)$ & $55.40(6.57)$ & $56.94(2.23)$ \\
\hline & Absolute & $220.06(10.32)$ & $197.17(5.13)$ & $15.82(0.56)$ & $83.29(0.69)$ & $77.68(1.00)$ & $38.35(4.80)$ & $53.79(1.34)$ \\
\hline & TUPE (a) & $220.78(8.49)$ & $201.86(6.14)$ & $3.56(0.64)$ & $61.11(12.33)$ & $58.59(11.18)$ & $13.75(3.21)$ & $34.25(6.03)$ \\
\hline & TUPE (r) & $212.54(3.02)$ & $192.31(9.32)$ & $5.00(1.46)$ & $72.05(11.82)$ & $66.01(9.39)$ & $33.61(10.73)$ & 44.17 (7.93) \\
\hline & Relative (k) & $184.79(6.02)$ & $165.70(6.85)$ & $5.56(1.40)$ & $72.94(3.63)$ & $69.32(5.43)$ & $14.18(5.07)$ & $40.50(2.83)$ \\
\hline & Relative (k/q) & $177.74(6.85)$ & $160.42(5.99)$ & $5.08(0.24)$ & $71.11(5.47)$ & $67.52(3.27)$ & $32.22(12.51)$ & $43.99(1.05)$ \\
\hline
\end{tabular}

Table 5: Full results (mean/std. over three seeds) 